\title{
Trends in Metal Oxide Stability for Nanorods, Nanotubes, and Surfaces
}

\author{
D. J. Mowbray ${ }^{12}$, J. I. Martínez ${ }^{13}$, F. Calle-Vallejo ${ }^{1}$, J. Rossmeisl ${ }^{1}$, K. S. Thygesen ${ }^{1}$, K. W. Jacobsen ${ }^{1}$, and J. K. Nørskov ${ }^{1 *}$ \\ ${ }^{1}$ Center for Atomic-scale Materials Design, \\ Department of Physics, Technical University of Denmark, \\ DK-2800 Kgs. Lyngby, Denmark \\ ${ }^{2}$ Nano-Bio Spectroscopy group and ETSF Scientific Development Centre, \\ Dpto. Física de Materiales, Universidad del País Vasco, \\ Centro de Física de Materiales CSIC-UPV/EHU-MPC and DIPC, \\ Av. Tolosa 72, E-20018 San Sebastián, Spain \\ ${ }^{3}$ Dpto. de Física Teórica de la Materia Condensada, \\ Universidad Autónoma de Madrid, E-28049 Madrid, Spain
}

(Received 17 December 2009)

\begin{abstract}
The formation energies of nanostructures play an important role in determining their properties, including the catalytic activity. For the case of 15 different rutile and 8 different perovskite metal oxides, we find that the density functional theory (DFT) calculated formation energies of $(2,2)$ nanorods, $(3,3)$ nanotubes, and the $(110)$ and (100) surfaces may be described semi-quantitatively by the fraction of metal-oxygen bonds broken and the bonding band centers in the bulk metal oxide.
\end{abstract}

PACS numbers: 73.22.-f 71.15.Mb 73.21.-b

The search for cleaner and more sustainable forms of energy provides a strong impetus to the development of more affordable, active, selective and stable new catalysts to convert solar radiation into fuels $[1,2]$. Just as the Haber-Bosch process fueled the population explosion of the $20^{\text {th }}$ century $[3,4]$, it is now hoped that new catalytic processes will provide sustainable energy in the $21^{\text {st }}$ century $[1,2]$.

Metal oxides are used extensively as catalysts, electrocatalysts, and photo-electrocatalysts [5-17]. One important property of oxides is their high stability in harsh oxidizing environments compared to their pure metal counterparts.

The structure of oxide nanoparticles may be determined by the surface energy. For instance, for $\mathrm{TiO}_{2}$ it has recently been shown that it is the surface energies which determine whether it takes the anatase or rutile structure at the nanoscale [18, 19]. Typically, oxide catalysts are in the form of nanoparticles or highly porous materials. The catalytic properties of these materials are determined to a high degree by the surface [20], and control of the surface structure will allow control of the reactivity [21-24]. However, formation energies of metal oxide surfaces are difficult to measure experimentally, and only a few values are available in the literature $[18,19]$. This makes the calculation of trends in surface and nanostructure energies an essential first step in understanding the properties of oxide catalysts, and the eventual design of novel catalytic materials.

In this study we provide DFT calculated $(2,2)$ nanorod, $(3,3)$ nanotube, and (110) surface formation energies for 15 different rutile metal oxides, along with the (100) surface formation energies for 8 different perovskite metal oxides [21]. Both the $(2,2)$ nanorod and $(3,3)$ nanotube structures are obtained by first rolling up a sheet of the material in the $\mathrm{PtO}_{2}$ structure. But due to its small diameter, the $(2,2)$ nanorod has a bonding structure resembling that of the bulk metal after relaxation, as shown in Fig. 1.

All DFT calculations have been performed with the plane wave code DACAPO using the RPBE exchange-correlation (a) $\mathrm{TiO}_{2}(2,2)$ Nanorod
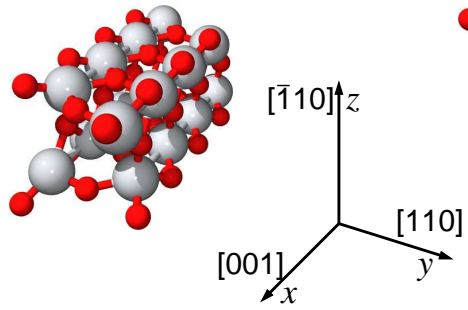

(c) $\mathrm{TiO}_{2}(110)$ Surface

(b) $\mathrm{TiO}_{2}(3,3)$ Nanotube
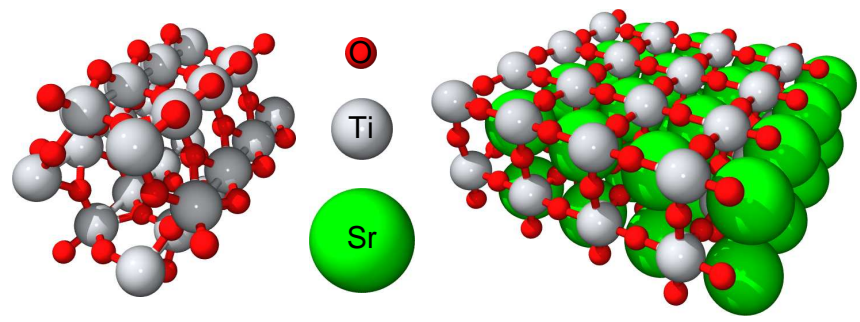

(d) $\mathrm{SrTiO}_{3}(100)$ Surface

FIG. 1: Structural schematics for (a) $\mathrm{TiO}_{2}(2,2)$ nanorod, (b) $\mathrm{TiO}_{2}$ $(3,3)$ nanotube, (c) $\mathrm{TiO}_{2}$ (110) surface and (d) $\mathrm{SrTiO}_{3}$ (100) $\mathrm{TiO}_{2}$ and $\mathrm{SrO}$ terminated surfaces. Axes and Miller indices for the rutile structures are shown for (a) and (c).

(xc)-functional [28, 29], converged plane wave cutoffs of 350 $\mathrm{eV}$ and $400 \mathrm{eV}$ for rutile and perovskite metal oxides respectively, along with a density cutoff of $500 \mathrm{eV}$. The occupation of the Kohn-Sham orbitals was calculated at $k_{B} T \approx 0.1 \mathrm{eV}$, with all energies extrapolated to $T=0 \mathrm{~K}$. The product of the supercell dimensions and the number of k-points [30] is $\gtrsim 25 \AA$ in all repeated directions. For the anti-symmetric perovskite surface slab calculations a dipole correction has been employed [31].

We have performed structural relaxations until a maximum force below $0.05 \mathrm{eV} / \AA$ was obtained. At the same time we 


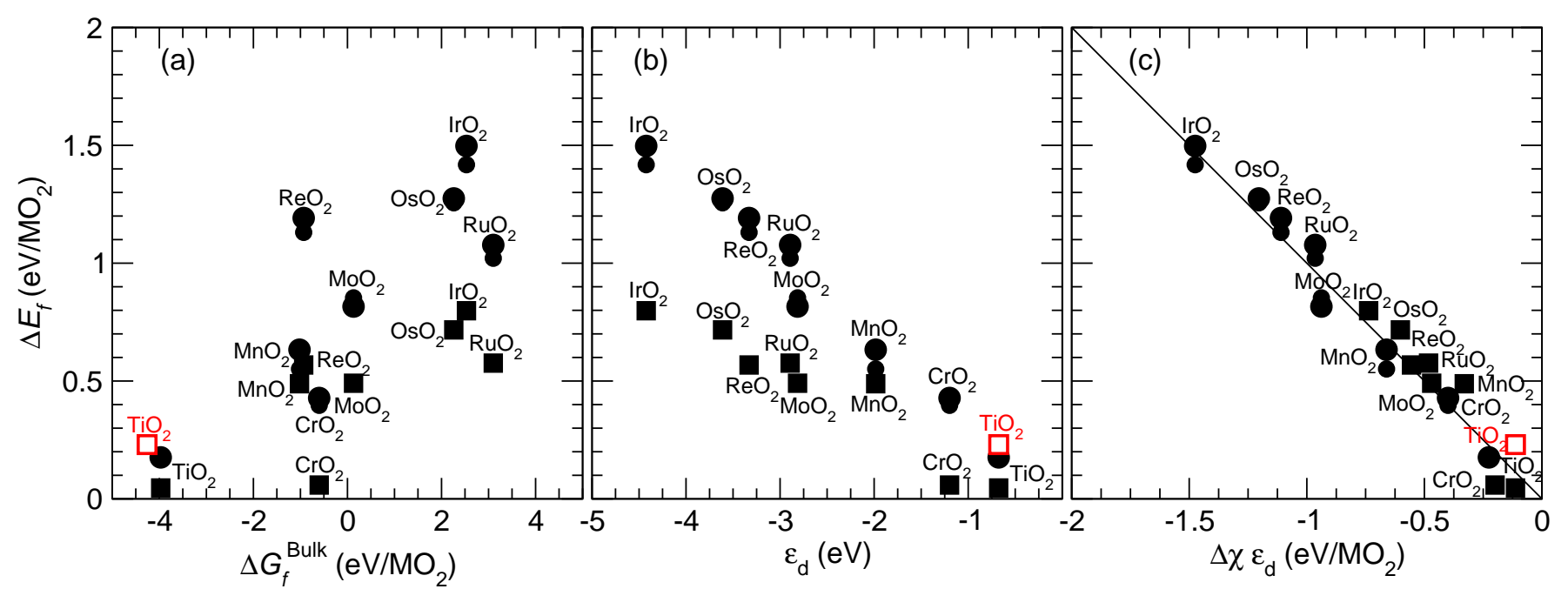

FIG. 2: Formation energy $\Delta E_{f}$ of rutile metal oxide $(2,2)$ nanorods $(\bullet),(3,3)$ nanotubes $(\bullet)$, and (110) surfaces $(\boldsymbol{\square})$ in eV/MO $/ \mathrm{Ms}_{2}$ vs. (a) bulk heat of formation $\Delta G_{f}^{\text {Bulk }}$ for rutile metal oxides in eV/MO $\mathrm{MO}_{2}$ from Ref. 25, (b) $d$-band center $\varepsilon_{d}$ for the bulk metal oxide relative to the Fermi energy in $\mathrm{eV}$, and (c) fraction of $\mathrm{M}-\mathrm{O}$ bonds broken times the $d$-band center $\Delta \chi \varepsilon_{d}$ in eV/MO $\mathrm{MO}_{2}$. The experimental nanoparticle surface energy [19] and bulk formation energy [26, 27] for rutile $\mathrm{TiO}_{2}(\square)$ are provided for comparison.

have minimized the strain on the unit cell in all periodically repeated directions, and employed more than $10 \AA$ of vacuum between repeated nanorods, nanotubes, and surface slabs. Schematics of the $\mathrm{TiO}_{2}(2,2)$ nanorod, $(3,3)$ nanorod, and (110) surface, along with the $\mathrm{SrTiO}_{3}(100) \mathrm{TiO}_{2}$ and $\mathrm{SrO}$ terminated surfaces [32] are shown in Fig. 1. The supercells used have been repeated four times along the nanorod and nanotube axis in Fig. 1(a) and 1(b), and four times in the surface plane for the surfaces shown in Fig. 1(c) and 1(d). For the $\mathrm{TiO}_{2}(110)$ surface our four layer thick slab yields surface formation energies of $0.44 \mathrm{~J} / \mathrm{m}^{2}$ in reasonable agreement with the GGA [33] 12 layer thick slab value of $0.50 \mathrm{~J} / \mathrm{m}^{2}$ [18], and the B3LYP [34] 9 layer thick slab value of $0.67 \mathrm{~J} / \mathrm{m}^{2}$ [35]. Differences amongst these values are attributable to the choice of xc-functional and number of layers, especially for uneven slab calculations, which tend to yield higher energies and converge slower [18]. As expected, these values are below the experimental nanoparticle surface energy of $2.2 \pm 0.2 \mathrm{~J} / \mathrm{m}^{2}$ for $\mathrm{TiO}_{2}$, as shown in Fig. 2 [19].

Spin polarized calculations have been performed only for $\mathrm{CrO}_{2}$ and $\mathrm{MnO}_{2}$ rutile metal oxides, while all perovskites have been calculated spin polarized. However, spin only proved to be important for the energetics of $\mathrm{CrO}_{2}, \mathrm{MnO}_{2}$, $\mathrm{SrCrO}_{3}, \mathrm{SrMnO}_{3}, \mathrm{SrFeO}_{3}, \mathrm{SrCoO}_{3}$, and $\mathrm{SrNiO}_{3}$. A table of these results is provided in Ref. 36.

Serious questions have been raised as to whether standard DFT calculatons can describe metal oxides with sufficient accuracy. For the purposes of the present study, it is worth noting that it has recently been demonstrated that DFT calculations do reproduce semi-quantitatively the formation energies of bulk rutile and perovskite metal oxides [25, 37].

The formation energies of the various surfaces and nanostructures from Fig. 1 are shown in Fig. 2. In the following we shall analyze the nature of the formation energies, and relate them to other characteristics of the materials. An obvious choice of descriptor for the (110) metal oxide surface formation energy $\Delta E_{f}^{(110)}$ might be the bulk heat of formation $\Delta G_{f}^{\text {Bulk }}$, which is well described by DFT for rutile metal oxides [25]. However, as shown in Fig. 2(a), these quantities appear to be poorly correlated. We instead observe a strong correlation between $\Delta E_{f}^{(110)}$ and the $d$-band center $\varepsilon_{d}$ for the bulk rutile metal oxides, as shown in Fig. 2(b). Here, $\varepsilon_{d}$ is the average energy, relative to the Fermi level $\varepsilon_{F}$, of the density of states (DOS) projected onto the metal's atomic $d$-orbitals in the metal oxide $n_{d}(\varepsilon)$, so that $\varepsilon_{d} \equiv \int\left(\varepsilon-\varepsilon_{F}\right) n_{d}(\varepsilon) d \varepsilon$ [38]. For the nanorod and nanotube formation energies, we also find little correlation with $\Delta G_{f}^{\text {Bulk }}$, but a strong correlation with $\varepsilon_{d}$.

This suggests metal oxide surface and nanostructure formation energies may be considered perturbations of the bulk metal oxide's electronic structure due to bond breaking. In this case $\Delta E_{f}$ should be well described by the fraction of metal-oxygen $(\mathrm{M}-\mathrm{O})$ bonds which are broken $\Delta \chi$, times the $\mathrm{M}-\mathrm{O}$ bond energy in the bulk $\varepsilon_{\mathrm{M}-\mathrm{O}}$. For the case of (110) surface formation $\Delta \chi^{(110)} \approx 1 / 6$ per $\mathrm{MO}_{2}$, while for nanorod and nanotube formation $\Delta \chi^{\mathrm{NT}} \approx 2 / 6 \approx 1 / 3$ per $\mathrm{MO}_{2}$. Assuming $\varepsilon_{\mathrm{M}-\mathrm{O}} \sim-\varepsilon_{d}$, we indeed find that $\Delta E_{f} \approx-\Delta \chi \varepsilon_{d}$, as shown in Fig. 2(c).

This correlation may be explained qualitatively by recalling that the $d$-band is most stable when the average energy of the DOS is $\varepsilon_{F}$, i.e. $\varepsilon_{d} \approx 0$. In most cases this is equivalent to having a half full $d$-band [38]. This is nearly the case for $\mathrm{TiO}_{2}$, where $\varepsilon_{d} \approx-0.67 \mathrm{eV}$, and the (110) surface becomes very stable $\left(\Delta E_{f}^{(110)} \approx 0.046 \mathrm{eV} / \mathrm{TiO}_{2}\right)$. As the $d$-band shifts down in energy, the energy stored in the $\mathrm{M}-\mathrm{O}$ bonds increases accordingly, so that $\varepsilon_{\mathrm{M}-\mathrm{O}} \sim-\varepsilon_{d}$.

A more detailed analysis based on the molecular orbitals 


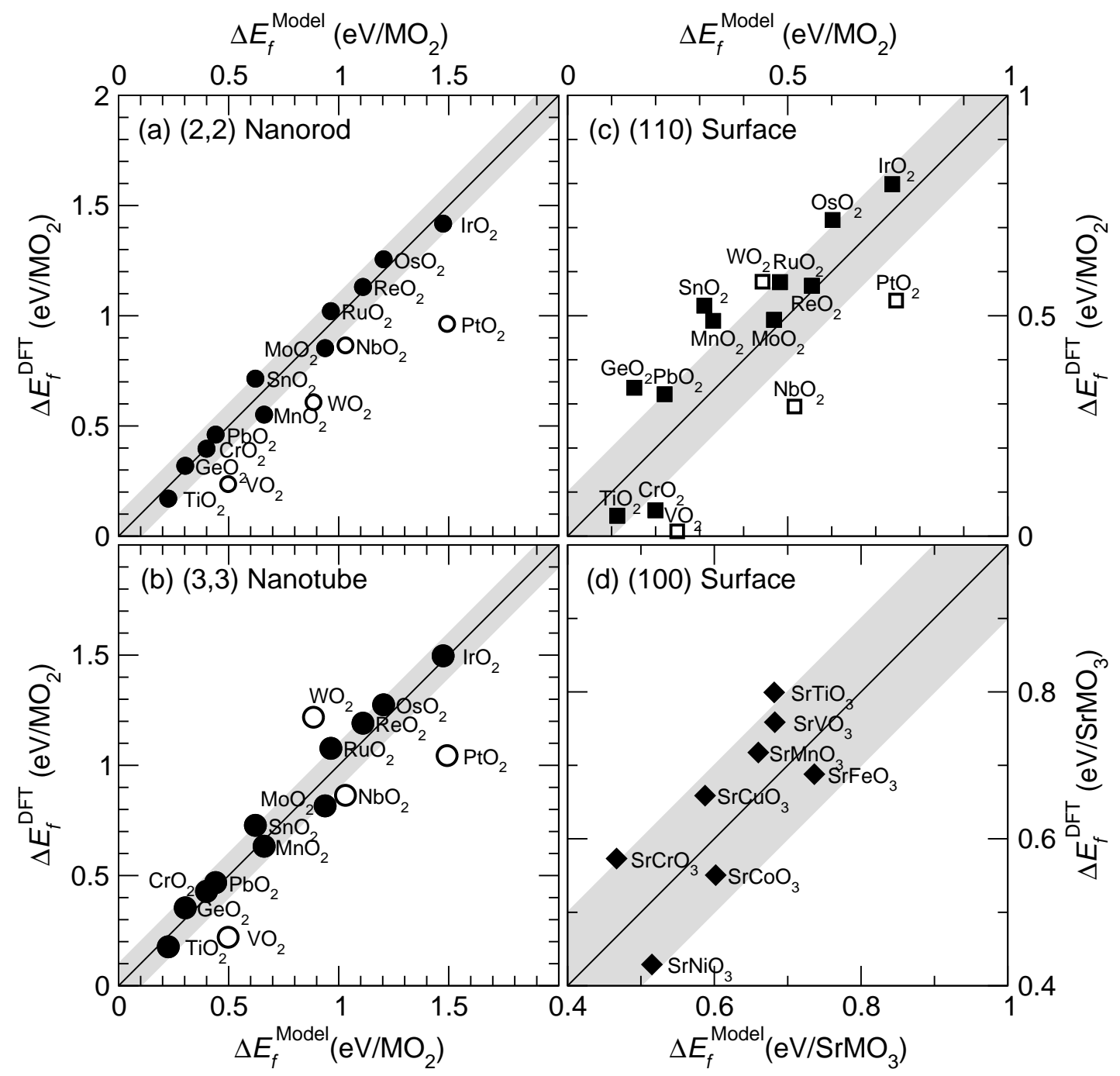

FIG. 3: DFT calculated formation energy $\Delta E_{f}^{\mathrm{DFT}}$ vs. model prediction $\Delta E_{f}^{\mathrm{Model}}$ for (a) $(2,2)$ nanorod $(\bullet)$, (b) $(3,3)$ nanotube $(\boldsymbol{\bullet})$, and (c) (110) surface $(\boldsymbol{\square})$ in $\mathrm{eV} / \mathrm{MO}_{2}$ for rutile and (d) (100) $\mathrm{MO}_{2}$ and SrO terminated surfaces $(\bullet)$ in $\mathrm{eV} / \mathrm{SrMO}_{3}$ for perovskites. Open symbols denote metal oxides whose most stable phase is not rutile. An error estimate of $\pm 0.1 \mathrm{eV}$ (grey region) is provided for comparison.

of the bulk metal oxide is presented in Refs. 36, 39-41. To summarize, as the number of $d$ electrons $N_{d}$ increases, the M$\mathrm{O}$ coordinately unsaturated (cus) bond becomes stronger. It is only this bond which is broken during (110) surface, nanorod and nanotube formation for rutile metal oxides. As shown in Ref. 36, this occurs as we progress from non-cus bonding $\left(N_{d}=2\right), \mathrm{M}-\mathrm{M}$ bond distortions $\left(N_{d}=3\right), \mathrm{M}-\mathrm{O}$ cus bonding $\left(N_{d}=4\right)$, non-bonding $\mathrm{M}$ orbitals with reduced distortions $\left(N_{d}=5,6\right)$, to stronger $\mathrm{M}-\mathrm{O} p_{\sigma}$ bonds $\left(N_{d}=7\right)$. Further, for a fixed number of $d$ electrons we also find that the bond strength increases as the DOS shifts down in energy. In effect, the energy stored in rutile metal oxide $\mathrm{M}-\mathrm{O}$ bonds is then equal to the energy cost for "shifting" the bonding metal orbitals so that their DOS is symmetric about the Fermi level. In such a case, both bonding with cus $\mathrm{O}$ atoms and distortions of the octahedral structure are minimized.

It is worth noting that we obtain near quantitative agree- ment between the DFT calculated formation energies and this simple model, when applied to naturally occurring transition metal rutile oxides. We now show how this model $\Delta E_{f}^{\text {Model }} \equiv-\Delta \chi \varepsilon_{d}$ may be extended to post transition metal oxides $\left(\mathrm{GeO}_{2}, \mathrm{SnO}_{2}\right.$, and $\left.\mathrm{PbO}_{2}\right)$ and $3 d$ transition metal perovskites $\left(\mathrm{SrMO}_{3}\right)$, as shown in Fig. 3 .

For post transition metals, the $d$-band is fully occupied, and does not participate in the $\mathrm{M}-\mathrm{O}$ bonding. Instead, the $\mathrm{M}-$ O bonding should occur via the occupied portion $(1 / 3)$ of the metal's $p$-band. In any case, we find that the $\mathrm{M}-\mathrm{O}$ bond energy $\varepsilon_{\mathrm{M}-\mathrm{O}}$ for post transition metal oxides is approximately negative one third the $p$-band center $-\varepsilon_{p} / 3$, so that $\Delta E_{f} \approx$ $-\Delta \chi \varepsilon_{p} / 3$. This approximation yields near-quantitative formation energies for post transition metal nanorods, nanotubes, and (110) surfaces, as seen in Fig. 3(a), (b), and (c), respectively.

Since perovskite metal oxides share the $\mathrm{MO}_{6}$ octahedral 
structure of rutile metal oxides, with interstitial $\mathrm{Sr}[42,43]$, it is reasonable to expect that the same correlation with the metal $d$-band centers $\varepsilon_{d}$ should hold. From the DFT calculations for the perovskite (100) surface, we obtain the average of the formation energies for both the $\mathrm{MO}_{2}$ and $\mathrm{SrO}$ terminated surfaces. As such, our model predicts $\Delta E_{f}^{(100)} \approx$ $-\left(\Delta \chi^{\mathrm{M}} \varepsilon_{d}^{\mathrm{M}}+\Delta \chi^{\mathrm{Sr}} \varepsilon_{d}^{\mathrm{Sr}}\right) / 2$, where $\Delta \chi^{\mathrm{M}} \approx 2 / 6 \approx 1 / 3$ per $\mathrm{SrMO}_{3}$ and $\Delta \chi^{\mathrm{Sr}} \approx 2 / 12 \approx 1 / 6$ per $\mathrm{SrMO}_{3}$. From Fig. 3(d), we find this is indeed the case.

In Fig. 3(a), (b), and (c) we also plot the DFT calculated formation energies versus $\Delta E_{f}^{\text {Model }}$ for transition metals whose most stable metal oxide phase is not rutile. For these compounds, denoted by open symbols in Fig. 3, we find our model typically overestimates formation energies.

For the HexABC layer structure of $\mathrm{PtO}_{2}$ [21] we find the formation energies are completely independent of $\varepsilon_{d}$ and $\varepsilon_{p}$, as shown in Ref. 36. However, this is also predicted by our model, since no $\mathrm{M}-\mathrm{O}$ bonds are broken $(\Delta \chi \approx 0)$ when forming the HexABC layer. Instead, the energy cost to form the HexABC layer is related to the preference of oxygen to be either $s p^{2}$ or $s p^{3}$ hybridized in the metal oxide.

In conclusion, we have demonstrated that the formation energies for nanotubes, nanorods, and surfaces of metal oxides may be determined semi-quantitatively from the fraction of $\mathrm{M}-\mathrm{O}$ bonds which are broken $\Delta \chi$ and the bonding band centers $\varepsilon_{d}$ and $\varepsilon_{p}$ in the bulk metal oxide. We anticipate such models will prove useful in predicting the formation energy of doped metal oxide surfaces and nanostructures, their reactant adsorption energies, and their activities.

The authors acknowledge financial support from the Danish Center for Scientific Computing and NABIIT. J.I.M. acknowledges the financial support of the STREP EU APOLLONB Project, and F.C.-V. acknowledges financial support from the Strategic Electrochemistry Research Center (SERC). The Center for Atomic-scale Materials Design (CAMD) is sponsored by the Lundbeck Foundation.

* Electronic address: norskov@fysik.dtu.dk

[1] J. Hemminger, G. Crabtree, and M. Kastner, eds., The Energy Challenges Report: New Science for a Secure and Sustainable Energy Future (Argonne National Laboratory, Argonne, Illinois, 2008).

[2] G. M. Whitesides and G. W. Crabtree, "Don't forget long-term fundamental research in energy", Science 315, 796 (Feb. 2007), doi:10.1126/science. 1140362 .

[3] V. Smil, "Detonator of the population explosion", Nature 400, 415 (Jul. 1999), doi:10.1038/22672 Millennium Essay.

[4] J. W. Erisman, M. A. Sutton, J. Galloway, Z. Klimont, and W. Winiwarter, "How a century of ammonia synthesis changed the world", Nat. Geosci. 1, 636 (Sep. 2008), doi:10.1038/ngeo325.

[5] J. Murphy, S. Srinivasan, and B. E. Conway, eds., Electrochemistry in Transition: From the 20th to the 21st Century (Plenum, New York, 1992).
[6] S. Trasatti, "Electrocatalysis in the anodic evolution of oxygen and chlorine", Electrochim. Acta 29(11), 1503 (1984), doi:10.1016/0013-4686(84)85004-5.

[7] A. Marshall, B. Brresen, G. Hagen, S. Sunde, M. Tsypkin, and R. Tunold, "Iridium oxide-based nanocrystalline particles as oxygen evolution electrocatalysts", Russ. J. Electrochem. 42(10), 1134 (Oct. 2006), doi:10.1134/S1023193506100223.

[8] E. Guerrini, H. Chen, and S. Trasatti, "Oxygen evolution on aged $\mathrm{IrO}_{x} / \mathrm{Ti}$ electrodes in alkaline solutions", J. Solid State Electrochem. 11(7), 939 (Jul. 2007), doi:10.1007/s10008-0060238-4.

[9] S. Chrétien and H. Metiu, " $\mathrm{O}_{2}$ evolution on a clean partially reduced rutile $\mathrm{TiO}_{2}(110)$ surface and on the same surface precovered with $\mathrm{Au}_{1}$ and $\mathrm{Au}_{2}$ : The importance of spin conservation", J. Chem. Phys. 129(7), 074705 (2008), doi:10.1063/1.2956506.

[10] J. Rossmeisl, Z.-W. Qu, H. Zhu, G.-J. Kroes, and J. Nørskov, "Electrolysis of water on oxide surfaces", J. Electroanal. Chem. 607(1-2), 83 (2007), doi:10.1016/j.jelechem.2006.11.008.

[11] Á. Valdés, Z.-W. Qu, G.-J. Kroes, J. Rossmeisl, and J. K. Nørskov, "Oxidation and photo-oxidation of water on $\mathrm{TiO}_{2}$ surface", J. Phys. Chem. C 112(26), 9872 (2008), doi:10.1021/jp711929d.

[12] A. Fujishima and K. Honda, "Electrochemical photolysis of water at a semiconductor electrode", Nature 238, 37 (1972), doi: $10.1038 / 238037 \mathrm{a} 0$.

[13] S. U. M. Khan, M. Al-Shahry, and W. B. Ingler, "Efficient photochemical water splitting by a chemically modified $\mathrm{n}-\mathrm{TiO}_{2}$ ", Science 297, 2243 (2002)

[14] M. R. Hoffmann, S. T. Martin, W. Y. Choi, and D. W. Bahnmann, "Environmental applications of semiconductor photocatalysis", Chem. Rev. 95, 69 (1995), doi:10.1021/cr00033a004.

[15] O. Khaselev and J. A. Turner, "A monolithic photovoltaicphotoelectrochemical device for hydrogen production via water splitting", Science 280(5362), 425 (Apr. 1998), doi:10.1126/science.280.5362.425.

[16] A. Heller, "Hydrogen-evolving solar cells", Science 223(4641), 1141 (Mar. 1984), doi:10.1126/science.223.4641.1141.

[17] M. Grätzel, "Photoelectrochemical cells", Nature 414(6861), 338 (2001)

[18] H. Perron, J. Vandenborre, C. Domain, R. Drot, J. Roques, E. Simoni, J.-J. Ehrhardt, and H. Catalette, "Combined investigation of water sorption on $\mathrm{TiO}_{2}$ rutile (110) single crystal face: XPS vs. periodic DFT”, Surf. Sci. 601, 518 (2007), doi:10.1016/j.susc.2006.10.015.

[19] M. R. Ranade, A. Navrotsky, H. Z. Zhang, J. F. Banfield, S. H. Elder, A. Zaban, P. H. Borse, S. K. Kulkaml, G. S. Doran, and H. J. Whitfield, "Energetics of nanorystalline $\mathrm{TiO}_{2}$ ", PNAS 99(2), 6476 (2001), doi:10.1073/pnas.251534898.

[20] J. Rogal and K. Reuter, Ab initio atomistic thermodynamics for surfaces: A primer. (Educational Notes RTO-EN-AVT-142, Neuilly-sur-Seine, 2007), ISBN isbn978-92-837-0057-9.

[21] D. J. Mowbray, J. I. Martínez, J. M. García Lastra, K. S. Thygesen, and K. W. Jacobsen, "Stability and electronic properties of $\mathrm{TiO}_{2}$ nanostructures with and without $\mathrm{B}$ and $\mathrm{N}$ doping", $\mathrm{J}$. Chem. Phys. 131, 014101 (2009), doi:10.1063/1.3148892.

[22] D. N. Tafen and J. P. Lewis, "Structure, stability, and electronic properties of thin $\mathrm{TiO}_{2}$ nanowires", Phys. Rev. B 80, 014104 (2009), doi:10.1103/PhysRevB.80.014104.

[23] A. Bandura and R. Evarestov, "From anatase (101) surface to tio2 nanotubes: Rolling procedure and first principles lcao calculations", Surface Science 603(18), L117 (2009), doi:10.1016/j.susc.2009.07.041. 
[24] D. V. Bavykin, J. M. Friedrich, and F. C. Walsh, "Protonates titanates and $\mathrm{TiO}_{2}$ nanostructured materials: Synthesis, properties, and applications", Adv. Mater. 18, 2807 (2006), doi:10.1002/adma.20050296.

[25] J. I. Martínez, H. A. Hansen, J. Rossmeisl, and J. K. Nørskov, "Formation energies of rutile metal dioxides using density functional theory", Phys. Rev. B 79, 045120 (2009), doi:10.1103/PhysRevB.79.045120.

[26] D. Lide, Handbook of Chemistry and Physics, 87th ed. (CRCPress, 2006-2007).

[27] M. Pourbaix, Atlas of Electrochemical Equilibria in Aqueus Solutions (Pergamon, London, 1966)

[28] B. Hammer, L. B. Hansen, and J. K. Norskov, "Improved adsorption energetics with density functional theory using revised Perdew-Burke-Ernzerhof functionals", Phys. Rev. B 59(11), 7413 (1999), doi:10.1103/PhysRevB.59.7413.

[29] S. R. Bahn and K. W. Jacobsen, "An object-oriented scripting interface to a legacy electronic structure code", Comput. Sci. Eng. 4(3), 56 (May 2002).

[30] H. J. Monkhorst and J. D. Pack, Phys. Rev. B 13, 5188 (1976), doi:10.1103/PhysRevB.13.5188.

[31] L. Bengtsson, "Dipole correction for supercell calculations", Phys. Rev. B 59(19), 12301 (1999), doi:10.1103/PhysRevB.59.12301.

[32] A. G. Schrott, J. A. Misewich, M. Copel, D. W. Abraham, and Y. Zhang, "A-site surface termination in strontium titanate single crystals", Appl. Phys. Lett. 79(12), 1786 (2001), URL http://link.aip.org/link/?APL/79/1786/1, doi:10.1063/1.1404129.

[33] J. P. Perdew and Y. Wang, "Accurate and simple analytic representation of the electron-gas correlation energy", Phys. Rev. B
45(23), 13244 (1992), doi:10.1103/PhysRevB.45.13244.

[34] A. D. Becke, "Density-functional thermochemistry. iii. the role of exact exchange", J. Chem. Phys. 98(7), 5648 (Apr. 1993), doi:10.1063/1.464913.

[35] A. Beltrán, J. Andrés, J. R. Sambrano, and E. Longo, "Density functional theory study on the structural and electronic properties of low index rutile surfaces for $\mathrm{TiO}_{2} / \mathrm{SnO}_{2} / \mathrm{TiO}_{2}$ and $\mathrm{SnO}_{2} / \mathrm{TiO}_{2} / \mathrm{SnO}_{2}$ composite systems", J. Phys. Chem. A 112 , 8943 (2008), doi:10.1021/jp801604n.

[36] See supplementary material for further details.

[37] J. I. Martínez, F. Calle-Vallejo, and J. Rossmeisl (2009), private communication.

[38] T. Bligaard and J. K. Nørskov, "Ligand effects in heterogeneous catalysis and electrochemistry", Electrochim. Acta 52, 5512 (2007), doi:10.1016/j.electacta.2007.02.0414.

[39] B. Jiang, J. M. Zuo, N. Jiang, M. O'Keeffe, and J. C. H. Spence, "Charge density and chemical bonding in rutile, $\mathrm{TiO}_{2}$ ", Acta Crystal. A 59, 341 (2003).

[40] D. B. Rogers, R. D. Shannon, A. W. Sleight, and J. L. Gillson, "Crystal chemistry of metal dioxides with rutilerelated structures", Inorg. Chem. 8(4), 841 (1969), doi:10.1021/ic50074a029.

[41] J. B. Goodenough, "Metallic oxides", Prog. Solid State Chem. 5, 145 (1971), doi:10.1016/0079-6786(71)90018-5.

[42] H. Yokokawa, Ann. Rev. Mater. Res. 33, 581 (Apr. 2003), doi:10.1146/annurev.matsci.33.022802.093856.

[43] Z. Cheng, S. Zha, L. Aguilar, and M. Liu, "Chemical, electri$\mathrm{cal}$, and thermal properties of strontium doped lanthanum vanadate", Solid State Ionics 176(23-24), 1921 (2005), doi:DOI: 10.1016/j.ssi.2005.05.009. 